\title{
Ensayos de propagación de algunas especies forestales aptas para el manejo de la microcuenca La Lejía, Cundinamarca, Colombia*
}

\author{
Propagation trials of some tree species suitablefor \\ rehabilitation of La Lejia microbasin, Cundinamarca, Colombia
}

Artículo recibido: febrero de 2011

Artículo aprobado: julio de 2011

Julieth Pérez-Vega

Leidy Acosta Rodríguez

Ángela Parrado-Rosselli*

\section{Resumen}

Se realizaron ensayos de propagación de 13 especies forestales, consideradas en estudios previos como aptas para la recuperación y manejo de la microcuenca La Lejía en el Municipio de Arbeláez, Cundinamarca, Colombia. Las especies estudiadas fueron la guadua (Guadua angustifolia Kunth), el madre de agua [Trichanthera gigantea (Humb. \& Bonpl)], el ocobo (Tabebuia rosea DC.), el nogal cafetero [Cordia alliodora (Ruiz \& Pav.) Cham.], el drago (Croton spp.), balú (Erythrina edulis Triana), el aguacate (Persea americana Mill.), el cedro de altura (Cedrela montana Turcz.), el cedro rosado (Cedrela odorata Griseb.), el caucho (Ficus spp.), el brevo (Ficus carica L.), la guayaba (Psidium guajava L.) y el tomate de árbol (Solanum betaceum Cav.). Para tal fin se instalaron tres viveros comunitarios en los que se evaluó la respuesta de la especie en uno de tres tipos de propagación (vegetativa: estacas; sexual: semillas y plántulas rescatadas del bosque) ante cinco diferentes tipos de sustrato:1) tierra de la finca, 2) tierra y abono orgánico, 3) tierra y cascarilla, 4) tierra y caldo microbiano y 5) tierra y cascarilla, abono, caldo microbiano. La respuesta se midió en términos del porcentaje de supervivencia/germinación, el cambio en el número de hojas y el cambio en la altura durante tres meses. Los resultados muestran que los tratamientos tierra con abono orgánico y tierra con cascarilla fueron significativamente mejores en términos de crecimiento y supervivencia para cualquiera de los tipos de reproducción evaluados. Las especies que mejor desempeño presentaron fueron el cedro de altura (Cedrela montana) y el balu (Erythrina edulis).Se resalta la importancia de los viveros para el éxito, monitoreo y seguimiento de estrategias de propagación de especies con algún valor para las comunidades locales.

Investigación Financiada por el Centro de Investigaciones y Desarrollo Científico - CIDC, Universidad Distrital Francisco José de Caldas.

** Integrantes del semillero de investigación "Saberes Campesinos en Medio Ambiente: Chicha y Maíz", adscrito al grupo Uso y Conservación de la Diversidad Forestal, Ingeniería Forestal, Facultad del Medio Ambiente y Recursos Naturales, Universidad Distrital Francisco José de Caldas. Correos de contacto: vikyjep@hotmail.com; leidy8502@hotmail.com; aparrador@udistrital.edu.co 


\section{Palabras clave}

Propagación de especies forestales, microcuenca La Lejía, Cundinamarca-Colombia

\section{Abstract}

Propagation trials were conducted on 13 tree species considered in previous studies as a suitable for rehabilitation of the La Lejiamicrobasin in Cundinamarca, Colombia. Studied species were Guadua angustifolia (guadua), Trichanthera gigantea (madre de agua), Tabebuia rosea (ocobo), Cordia alliodora (nogal cafetero), Croton spp (drago), Erythrina edulis (balú), Persea americana (aguacate), Cedrela montana (cedro de altura), Cedrela odorata(cedro rosado), Ficus spp (caucho), Ficus carica (brevo), Psidium guajava (guayaba) and Solanum betaceum (tomate de árbol). In three community nurseries we evaluated the response of seeds, rescued seedlings and hardwood cuttings in five substrate types (i.e. 1. soil, 2. soil and organic fertilizer, 3. soil and rice husk, 4. soil and a microbial mixture, and 5. all types combined). Species response was measured during three months in terms of germination/survival percentage and changes in leaf number and seedling height. The results showed that survival and growth were significantly higher in soil and organic fertilizer and soil and rice husk for any propagation type. The best performance was exhibited by Cedrela Montana and Erythrinaedulis. We highlight the importance of community nurseries for successful monitoring and implementation of propagation strategies of valuable tree species for local communities.

\section{Keywords}

Propagation of forest species, La Lejía Microbasin, Cundinamarca- Colombia

\section{Introducción}

Uno de los factores que más influye en la regulación hídrica de las cuencas es la presencia de cobertura vegetal (CENICAFÉ, 1975; Farrill, et al.,2 008). Esta permite mejorar el ciclo hidrológico, ayuda a controlar la cantidad y temporalidad del flujo del agua, al proteger los suelos de ser arrastrados y por ende evita la sedimentación y degradación de los ríos y la pérdida de fertilidad en las laderas (Salas, 2003). Además, favorece la infiltración y evita la escorrentía superficial directa, gracias a la producción de una capa de hojarasca sobre el suelo mineral y a la disposición de su sistema radicular, lo cual disminuye la velocidad e impacto de caída de la gota (Villegas, 2004; Farrill, et al., 2008).

En este sentido, existen algunas especies arbóreas que -a partir de diferentes estudios- han sido sugeridas para ser utilizadas en programas de conservación de suelos y manejo de cuencas (Otero, et al., 2006; Pérez-Vega \& Acosta, 2010), gracias a sus características fisiológicas, por ejemplo: la capacidad de fijación de nitrógeno, una alta producción de materia orgánica, su copa frondosa y su buena capacidad de enraizamiento, entre otras.

Teniendo en cuenta lo anterior, el objetivo de esta investigación fue el de realizar ensayos depropagación de 13 especies forestales, seleccionadas a partir de un trabajo previo por Pérez-Vega \& Acosta (2010), en 3 viveros comunitarios ubicados en la microcuenca La Lejía, del Municipio de Arbeláez, en Cundinamarca. Para tal fin, se evaluó la supervivencia y el desempeño en términos del cambio de altura y número de hojas, en función del tipo de sustrato utilizado para tres diferentes métodos de propagación: reproducción vegetativa, semillas, rescate de plántulas. Este trabajo se realizó dado el escaso conocimiento existente sobre la propagación de las especies en la zona, ante lo cual se hacenecesario conocer sus requerimientos de propagación y manejar un vivero local que supla la demanda de material vegetal a utilizar en un programa de manejo y recuperación de la cobertura vegetal, alrededor de la microcuenca.

\section{Materiales y métodos}

\section{Área de estudio}

El estudio se llevó a cabo en las veredas San Antonio (Sector La Lajita) y San Miguel (Sectores Las Rositas, El Club, y Tenerife), que pertenecen a la microcuenca La Lejía, ubicada en el municipio de Arbeláez $\left(4^{\circ} 15^{\circ} 23^{\circ} \mathrm{N} ; 74^{\circ} 29^{\circ} 43^{\circ} \mathrm{W}\right)$, Cundinamarca, Colombia. La zona, se encuentra ubicada entre 
los 1400 y $2750 \mathrm{~m}$ de altitud y corresponde a los ecosistemas naturales de bosque subandino y bosque andino muy húmedo. Pertenece al Orobioma Subandino de la Cordillera Oriental, y este a su vez al Orobioma del zonobioma húmedo tropical (IAVH 2007). La temperatura media es de $22^{\circ} \mathrm{C}$, la precipitación está alrededor de los $1650 \mathrm{~mm}$ al año, poseeun régimen bimodal con una mayor cantidad de lluvias en los meses de febrero a abril y de octubre a diciembre (CENICAFÉ, 2006).

Se instalaron tres viveros comunitarios, uno en el sector de La Lajita, otro en el sector de Las Rositas, y el último en el sector de El Club; en ellos los habitantes locales aportaron su conocimiento sobre el manejo de agricultura orgánica y recolección del material vegetal. En reuniones previas se acordó, en consenso, que el dueño del predio en donde se construyó el vivero era el responsable principal del mismo, pero también se nombraron personas responsables de cada familia para que se compartiera su cuidado, las labores de riego, la poda de malezas y el monitoreo del crecimiento.

\section{Especies en estudio}

De 19 especies priorizadas por Pérez-Vega y Acosta (2010) para ser utilizadas en la recuperación de la microcuenca La Lejía, se seleccionaron de forma concertada y con base en la disponibilidad de material vegetal (por la época del año), 13 especies (Tabla 1). Aunque algunas exhibenmás de un uso, así, por ejemplo, cinco especies son utilizadas principalmente como alimento y seis más son identificadas por la comunidad como "hidrorreguladoras", además de sus otros usos (Pérez-Vega \& Acosta, 2010).

\section{Toma de registros y análisis}

Para cada una de las especies seleccionadas se realizaron ensayos de reproducción sexual o asexual (reproducción por semilla, estacas, y rescate de plántulas), y un solo tipo de reproducción para cada especie (dependiendo de si ésta estaba o no en época reproductiva).A partir de allí se evaluó la respuesta de los individuos a cinco diferentes tipos de sustrato (Tabla 2).
Seis especies se propagaron por medio de semilla, cinco por medio de estacas y dos por medio de plántulas directamente colectadas en el bosque. Los sustratos utilizados fueron: 1) tierra de finca (de aquí en adelante tierra), el cual se consideró como control, 2) tierra y abono orgánico, 3) tierra y cascarilla, 4) tierra y caldo microbiano y 5) una combinación de todos los tratamientos: tierra, abono orgánico, cascarilla y caldo microbiano (Tabla 2).

Posteriormente se evaluó el porcentaje de supervivencia de la regeneración natural (plántulas del bosque) y de la reproducción vegetativa (estacas que presentaron enraizamiento y yemas nuevas en los primeros 35 días), y el porcentaje de germinación y supervivencia en el caso de la reproducción sexual. Se cuantificó el número de hojas nuevas y las alturas totales de cada uno de los individuos. Los datos se tomaron a los 35, 60 y 90 días, a partir del establecimiento del ensayo. A los datos de número de hojas nuevas y alturas finales a los 90 días, se les realizaron pruebas de normalidad (Shapiro - Wilk). Aquellos que no presentaron una distribución normal se transformaron por el método Box- Cox. En caso que el $\lambda$ hallado en la transformación fuese igual a 1 , los datos no se normalizaron. Se realizó un Análisis de varianza de una vía (ANOVA) para establecer si existieron diferencias significativas entre los tratamientos. En el caso de existir diferencias, se realizó una prueba de comparación múltiple de Tukey. Los ensayos que no se ajustaron a una distribución normal, se analizaron por medio de una prueba de Kruskall-Wallis para evaluar la hipótesis de igualdad entre las medianas de los tratamientos.

\section{Resultados}

\section{Propagación vegetativa}

El porcentaje de sobrevivencia para el drago (Croton spp.) y el caucho (Ficus spp.) fue de cero en la primera medición (35 días), por lo que no se incluye en los análisis posteriores. Para el brevo ( $F i-$ cus carica), se encontraron diferencias significativas en el desempeño entre tratamientos (Tabla 3), siendo la combinación de tierra y caldo microbiano la que mayor porcentaje de supervivencia, nú- 
mero de hojas nuevas y cambios en altura presentó (Figura 1,Tabla 3), mientras que el menor desempeño se presentó en la tierra con abono orgánico. La prueba post hoc mostró diferencias significativas entre tierra y caldo microbiano con todos los demás tratamientos.

Para el Madre de agua (Trichanthera gigantea), la tierra con abono orgánico fue el tratamiento que generó,significativamente, los mayores porcentajes de supervivencia, número de hojas nuevase incremento en altura (Figura 1, Tabla 3). En contraste, el mejor desempeño para la guadua (Guadua angustifolia) se presentó en la tierra con cascarilla(Figura 1, Tabla 3). No obstante, esta no mostró diferencias significativas con el tratamiento control (tierra de la finca, post hoc de Tukey, Tabla 3).

\section{Propagación por semilla}

El porcentaje de sobrevivencia para el tomate de árbol (Solanum betaceum Cav.)fue de cero en la primera medición (35 días), por lo que no se incluye en los análisis posteriores.En los ensayos del nogal cafetero se encontraron diferencias significativas entre los tratamientos, en donde la supervivencia, el número de hojas nuevas y el cambio en la altura fue mayor en la tierra con abono orgánico (Figura 2, Tabla 4), siendo éste significativamente diferente a todos los demás tratamientos (Post-hoc Tuckey). Para el ocobo (Tabebuia rosea), el tratamiento más efectivo en germinación, número de hojas y alturas fue la tierra con abono orgánico, el cual sólo mostró diferencias significativas con la tierra con caldo microbiano (Tabla 4). Para guayaba (Psidium guajava), el tratamiento de tierra de la finca (control) fue el que tuvo el mayor porcentaje de sobrevivencia y número de hojas nuevas(Figura 2, Tabla 4), mientras que el mayor cambio en la altura se presentó para la tierra con abono orgánico (Tabla 4). En contraste, el tratamiento que menor desempeño mostró fue la tierra con cascarilla.

En el ensayo de propagación por semilla del aguacate (Persea americana) se observó que el mejor desempeño en supervivencia fue en el tratamiento de tierra y abono orgánico, seguido del tratamiento de tierra y cascarilla; en ellos se encontró, a través de la prueba Post-hoc, que los dos tuvieron diferencias significativas con todos los demás tratamientos para número de hojas y alturas (Figura 2, Tabla 4). De la misma forma, el balu (Erythrina edulis) presentó el mejor desempeño en supervivencia en el tratamiento de tierra y abono orgánico y el posthoc mostró que este tratamiento y el tratamiento control (tierra), presentaron los mejores resultados en número de hojas y alturas frente a los otros (Figura 2, Tabla 4).

\section{Regeneración natural}

En la Figura 3 y en la Tabla 5 se pueden observar los resultados de los ensayos de cedro de altura (Cedrela montana), éstos muestran que la tierra con cascarilla fue la más efectiva en el desempeño de las plantas, en términos del porcentaje de supervivencia,número de hojas nuevas, y cambio en la altura. Asimismo, este tratamiento mostró diferencias significativas con todos los demás tratamientos, excepto con la tierra y caldo microbiano (Tabla 5). En contraste, y a pesar de ser el mismo género, encedro rosado (Cedrela odorata) la tierra con abono orgánico obtuvo el mejor desempeño en las variables anteriormente descritas (Figura 3, Tabla 5), seguido del tratamiento control (tierra).

\section{Discusión de resultados}

En primer lugar, los resultados de este estudio muestran que, para la mayoría de las especies estudiadas, los tratamientos tierra con abono orgánico y tierra con cascarilla fueron significativamente mejores en términos de crecimiento y supervivencia para cualquiera de los tipos de reproducción evaluados. La importancia de estos dos tratamientos, posiblemente, radica en que estos son ricos en fósforo y nitrógeno, elementos fundamentales en los inicios del ciclo de la vida de las plantas, pues son utilizados principalmente para formar estructuras radiculares y de crecimiento (Trujillo, 2003). Adicionalmente, según Arriaga, et al. (1994), el tratamiento con cascarilla favorece la porosidad y, cuando la propagación espor semilla, permite la expansión de las raíces, lo cual parece ser el caso para la guadua y el cedro de altura. 
Sería interesante evaluar de forma más profunda, en el presente ensayo, las especies que presentaron una alta o total mortalidad. Por otro lado, la propagación vegetativa ha sido una técnica muy útil para una gran cantidad de especies de plantas, por lo que es sorprendente que el drago (Croton spp.) y el caucho (Ficus spp.) mostraron un alto porcentaje de mortalidad; por lo anterior, valdría la pena realizar un nuevo ensayo en donde se evalúen más variables. Del mismo modo que debe hacerse para la reproducción por semilla en el caso del tomate de árbol (Solanum betaceum), realizar estudios que evalúen la necesidad de tratamientos pregerminativos, la estación de fructificación de la especie y la calidad de la cosecha, podrían ser útiles en futuras estrategias de propagación para esta especies.

En segundo lugar, la especie que mejor desempeño presentó,en términos de supervivencia, cambio en la altura y del número de hojas, durante el desarrollo del estudio fue Cedrela montana. Ésta ha sido reportada en la literatura como una especie que presenta una alta resistencia en diferentes ambientes y circunstancias (Urgiles, et al., 2009; Webber, et al., 2008). La especie que le siguió en porcentaje de supervivencia fue el balú (Erythrina edulis), mientras que las demás 11 especies presentaron porcentajes de supervivencia menores al $70 \%$, en cualquiera de los tratamientos. No obstante, es importante tener en cuenta que el balú únicamente presentó porcentajes de supervivencia mayores al $80 \%$, similar a lo reportado en la literatura (Acero, 2002), para el tratamiento de tierra y abono orgánico, lo que ratifica la importancia de este tratamiento en la germinación.

Aunque no se tienen registros continuos de supervivencia después de los 90 días, desde el establecimiento de los ensayos, los pobladores locales manifiestan que -a pesar de estar en vivero- las plantas se ven altamente afectadas por las condiciones climáticas; por ejemplo, mencionan que pasados los 90 días del establecimiento el porcentaje de supervivencia del brevo (Ficus carica) disminuyó notoriamente, al igual que el aguacate (Persea americana) y el cedro rosado (Cedrela odorata).
Por lo tanto, estudios más amplios en el tiempo son importantes para evaluar el desempeño de las plántulas en estadios posteriores y durante el primer año después de la germinación.

Finalmente es de resaltar la importancia de incluir herramientas y mecanismos de participación en este tipo de trabajos, tales como la creación de viveros comunitarios, pues genera apropiación por parte de la comunidad de la zona, y trascendencia en el tiempo de este tipo de estrategias; además, permite un continuo seguimiento y cuidado del material a propagar yse mantiene el material vegetal necesario para la reforestación y enriquecimiento vegetal de zonas prioritarias de manejo.

\section{Conclusiones}

- El desempeño de las 13 especies estudiadas-medido en términos de supervivencia, cambio en la altura y número de hojas- fue significativamente mayor en los tratamientos: tierra y abono orgánico; tierra con cascarilla.

- La especie que mejor desempeño presentó durante el desarrollo del estudio fue Cedro rosado (Cedrela montana.); sin embargo, no se pueden hacer comparaciones directas con las otras especies puesto que a cada una de ellas se le evaluó un solo tipo de reproducción.

- La propagación de especies de importancia localdebe hacerse con base en las condiciones de cada sector y con un monitoreo permanente por parte de las comunidades locales.

\section{Agradecimientos}

Agradecemos al Centro de Investigaciones y Desarrollo Científico (CIDC) por el apoyo financiero, a través de la convocatoria 004 de 2008 para semilleros de investigación. Gracias también a la Administración Municipal de Arbeláez, Cundinamarca y a las comunidades locales de las veredas San Antonio y San Miguel por su colaboración en la etapa de campo. A Max Triana y a Luis Fernando Ortiz, por su revisión y comentarios críticos al proyecto y a versiones previas de este artículo. 


\section{Bibliografía}

Acero, L. (2002). Guía para el cultivo y aprovechamiento del Chachafruto (Erythrina edulis Triana). [Segunda edición]. Colombia: Convenio Andrés Bello.

Arriaga, V., Cervantes, V. \& Vargas, A. (1994). Manual de reforestación con especies nativas. México, D. F.: SEDESOL. Instituto Nacional de ecología UNAM. Facultad de Ciencias.

CENICAFÉ (1975). Manual de conservación de suelos de ladera. Chinchiná, Colombia.

CENICAFE (2006). Anuario Metereologico Cafetero 2006. Chinchiná, Colombia: Centro Nacional de Investigaciones de Cafe "Pedro Uribe Mejia", Federacion Nacional de Cafeteros de Colombia.

Farrill, A.,Suarez, T. \&Valle, M. (2008). "Recuperación del bosque de galería en la submicrocuenca nacimiento del Río Máximo". En Revista Forestal Baracoa, vol. 27, núm. 2. Cuba, La Habana: Instituto de Investigaciones Forestales.

IAVH-Instituto de investigación en Recursos Biológicos Alexander von Humboldt. (2007). Mapa de ecosistemas Andinos de Colombia. Instituto de Investigaciones Alexander Von Humboldt[en línea].Disponible en:http://hermes.humboldt.org.co/ecosistemas/andes/

IDEAM. (2006). Atlas climatológico de Colombia. Bogotá: Imprenta Nacional de Colombia.

Otero, J., Suarez, L., Quiceno, M. \& Cabrera, E. (2006). "Caracterización, uso, y manejo de cercas vivas en agroecosistemas ganaderos de montaña en Colombia”. En Lyonia vol. 10, núm. 2,pp. 117-136.
Pérez, J. \& Acosta, L. (2010). Selección participativa de especies forestales aptas para la recuperación, conservación y manejo de la microcuenca La Lejía, Cundinamarca, Colombia [Trabajo de grado en Ingeniería Forestal].Universidad Distrital Francisco José de Caldas.

Salas, J. (2003). “Determination of optimal water head pressure in water distribution network methodology".World Water \& Environmental Resources Congress 2003 \& Related Symposia. American Society of Civil Engineers ASCE.

Trujillo, E. N. (2003). Manual de árboles[segunda edición]. Bogotá, Colombia: Ediciones El Semillero.

Urgiles, N., Loján, P., Aguirre, N., Blaschke, H., Günter, S., Stimm, B.\& Kottke, I. (2009). "Application of mycorrhizal roots improves growth of tropical tree seedlings in the nursery: a step towards reforestation with native species in the Andes of Ecuador". En New Forest,vol 38, núm. 3, pp. 229-239.

Villegas, J. C. (2004). "Análisis del conocimiento en la relación agua-suelo-vegetación para el Departamento de Antioquia”. En Revista EIA,núm.1, pp. 73-79.

Webber, M., Günter, S., Aguirre, N., Stimm, B.\&Mosandl, R. (2008). "Reforestation of abandoned pastures: silvicultural means to accelerate forest recovery and biodiversity" En: Gradients in a tropical mountain ecosystem of Ecuador, E. Beck, J. Bendix, I. Kottke, F. Makeschin, \& R. Mosandl, (edits.). Berlín: Springer-Verlag. 


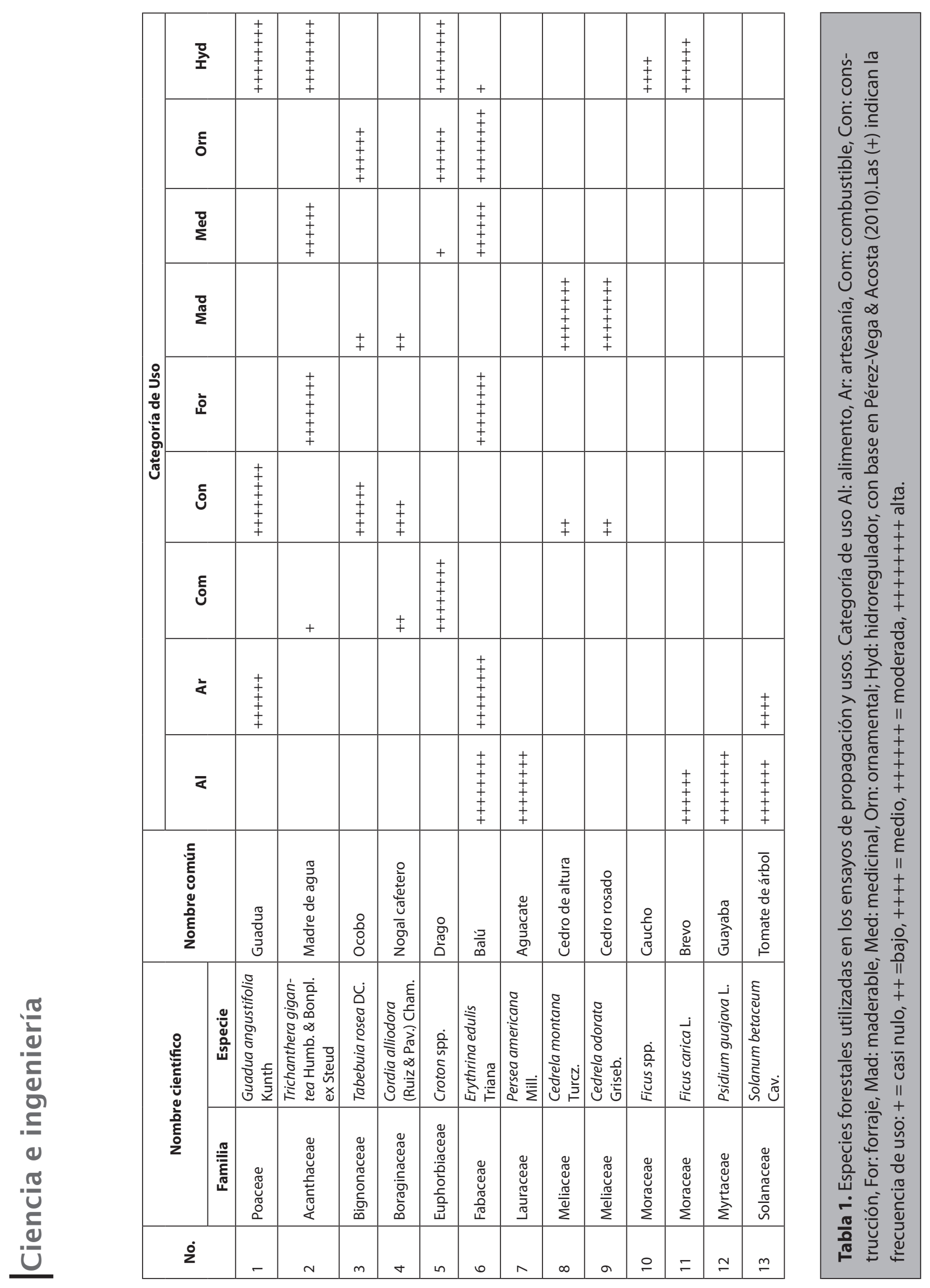




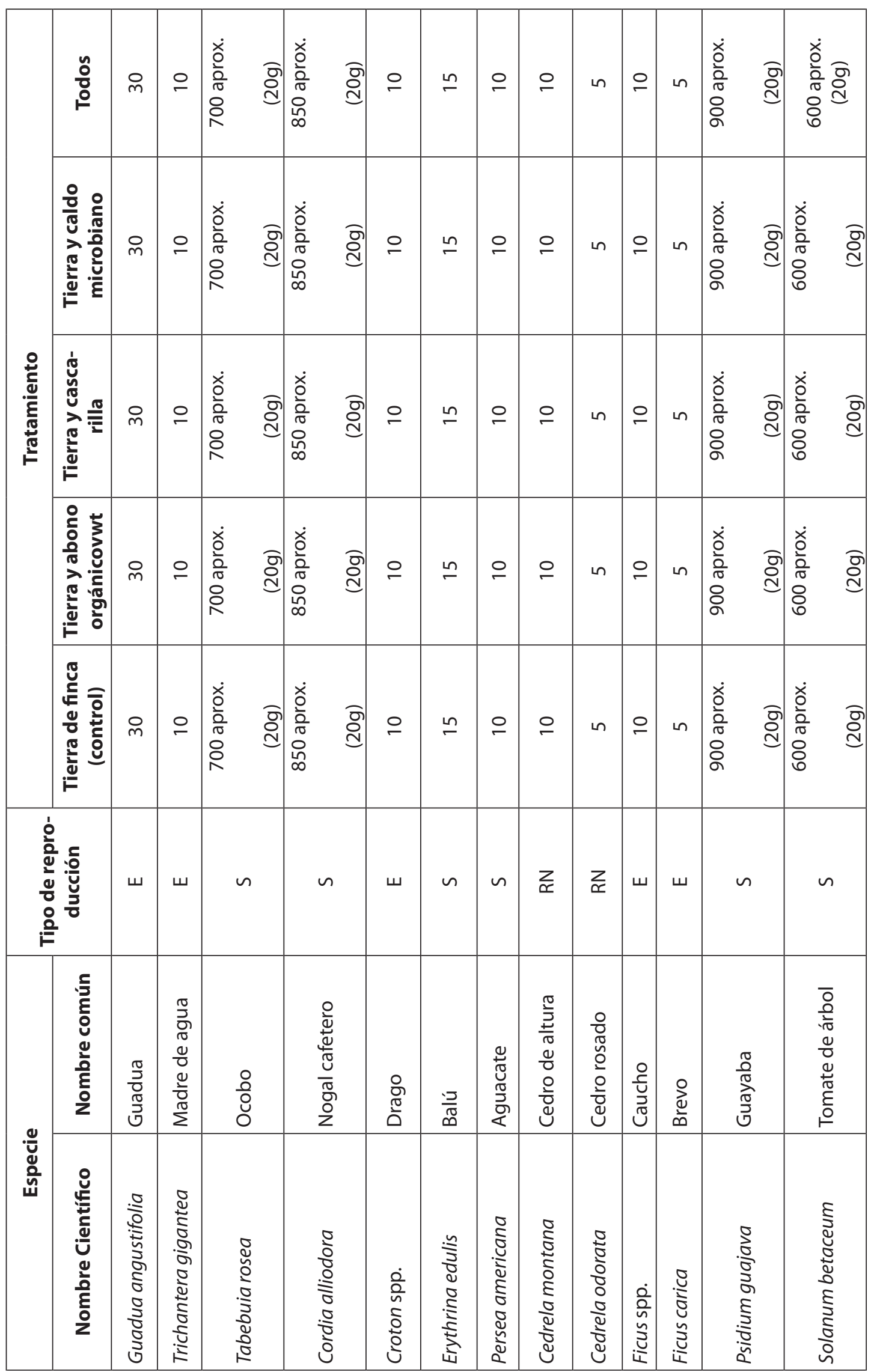

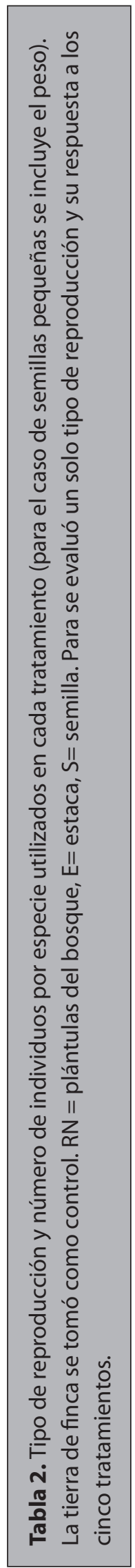

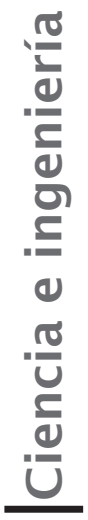

REVISTA CIENTÍFICA / ISSN 0124 2253/ JULIO - DICIEMBRE DE 2011 / No. 14 / BOGOTÁ, D.C 


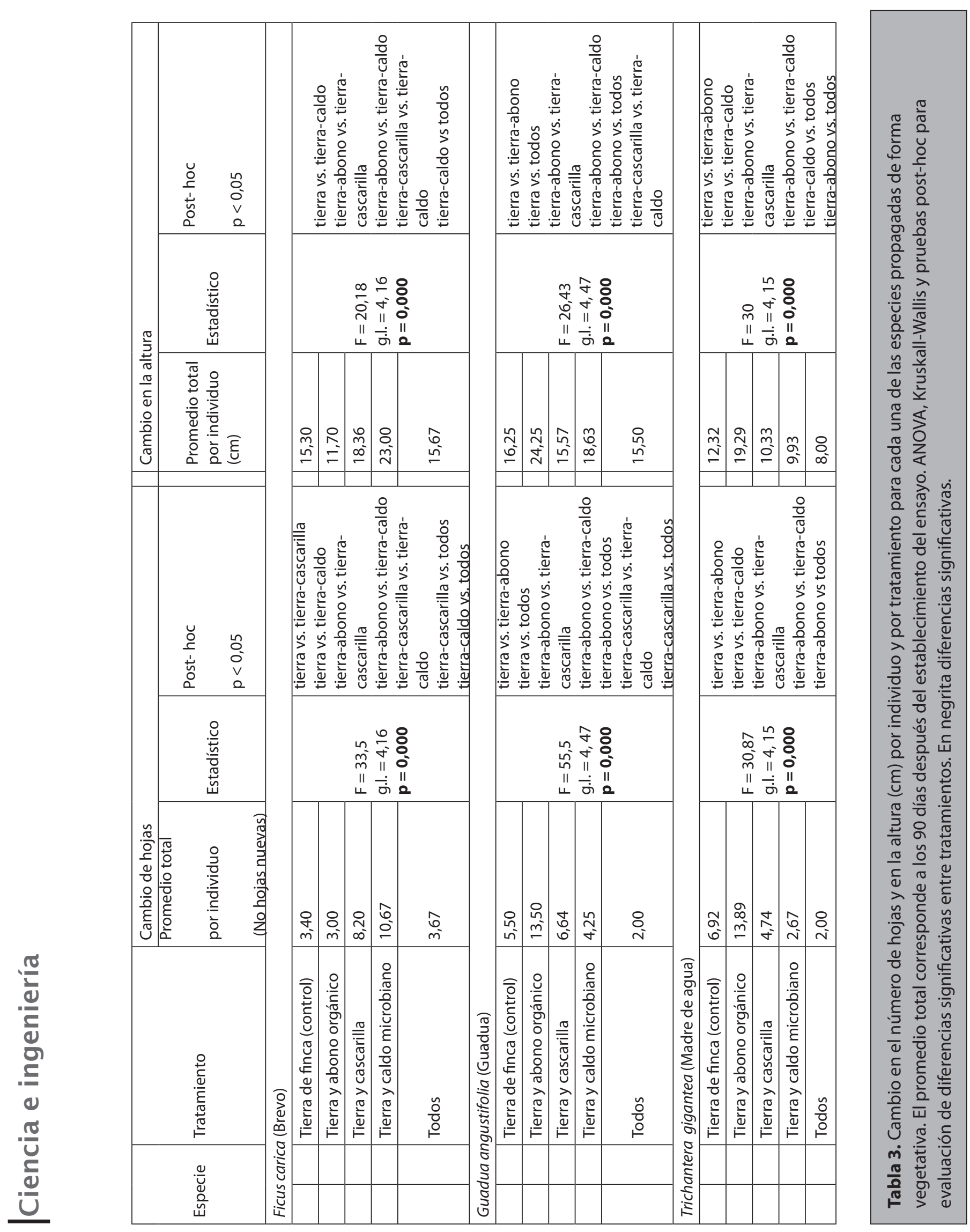




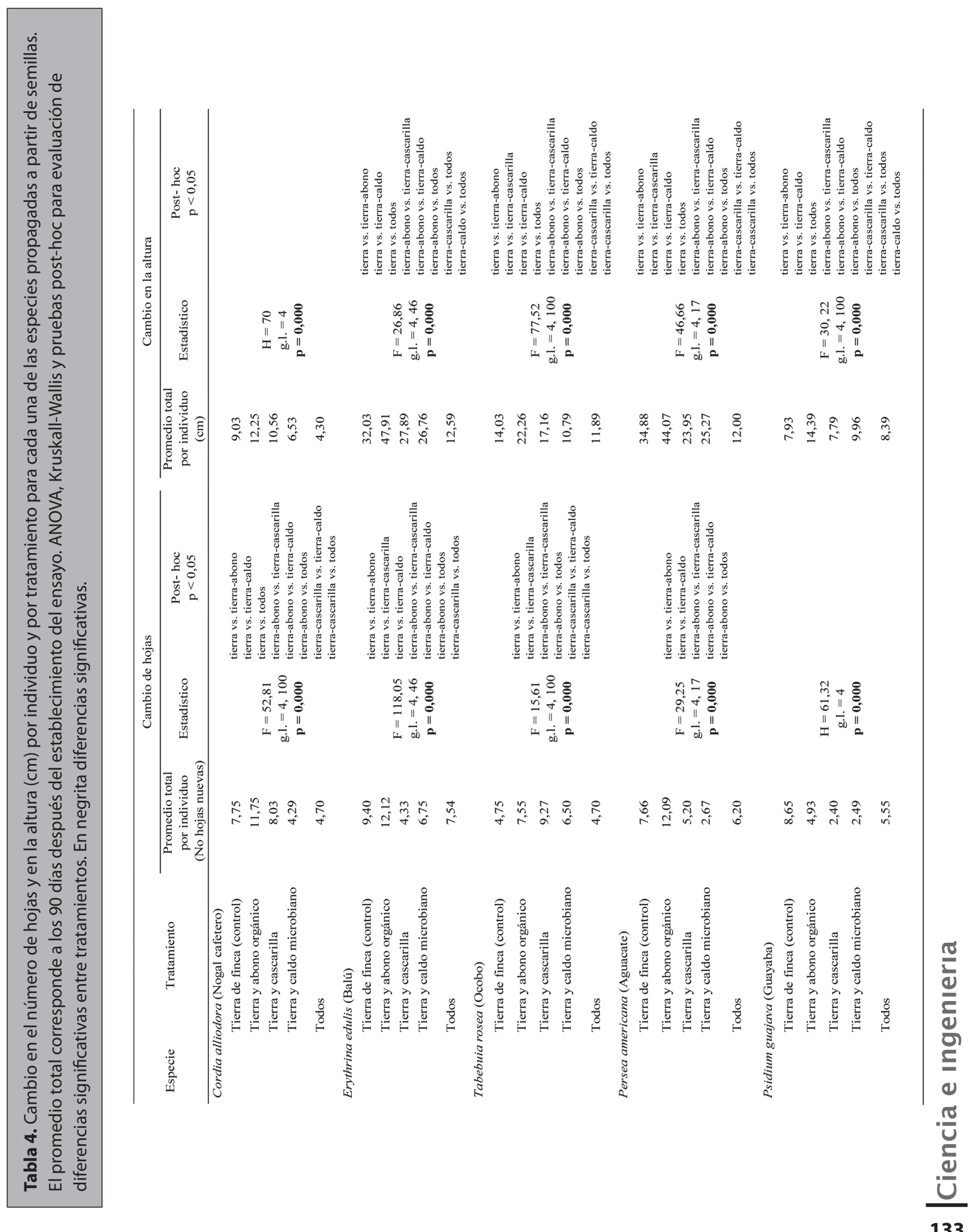

REVISTA CIENTÍFICA / ISSN 0124 2253/ JULIO - DICIEMBRE DE 2011 / No. 14 / BOGOTÁ, D.C. 


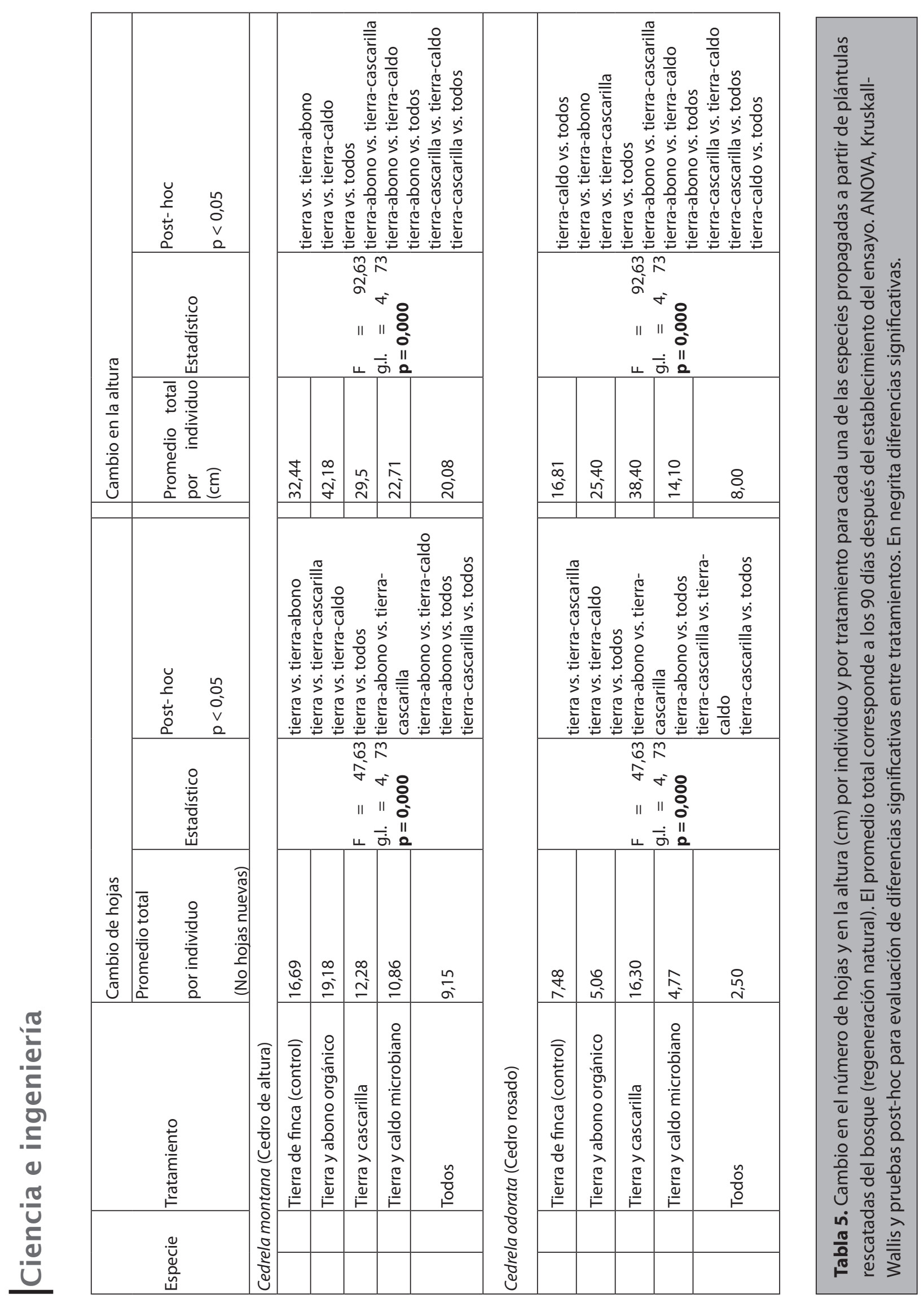

REVISTA CIENTÍFICA / ISSN 0124 2253/ JULIO - DICIEMBRE DE 2011 / No. 14 / BOGOTÁ, D.C. 

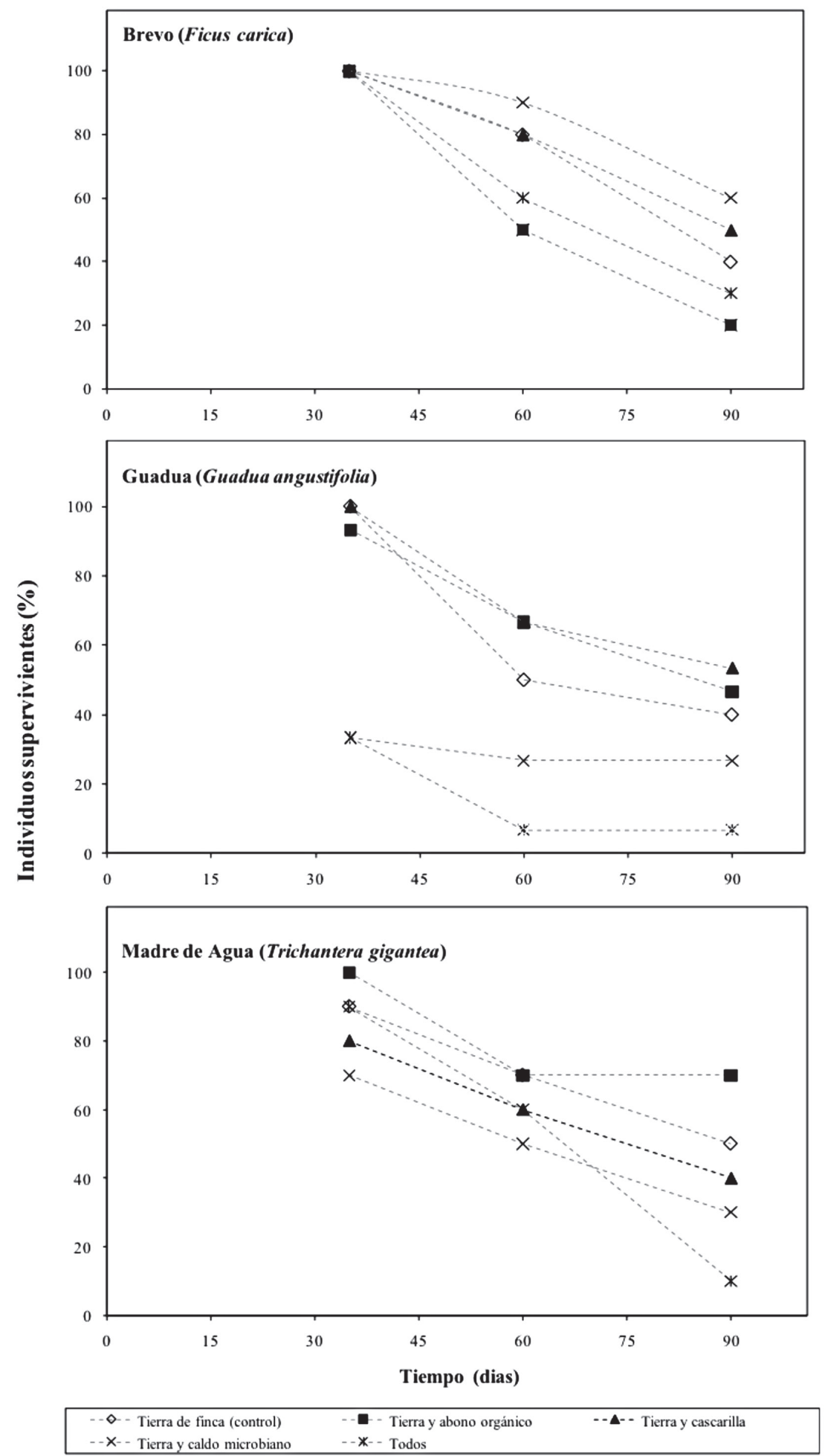

Figura 1. Porcentaje de supervivencia en los ensayos de propagación vegetativa de brevo (Ficus carica $\mathrm{N}=25$ ); guadua (Guadua angustifolia $\mathrm{N}=150$ ); y madre de agua (Trichanthera gigantea $\mathrm{N}=50$ ) durante 90 días desde el establecimiento del ensayo.

Fuente: elaboración propia 

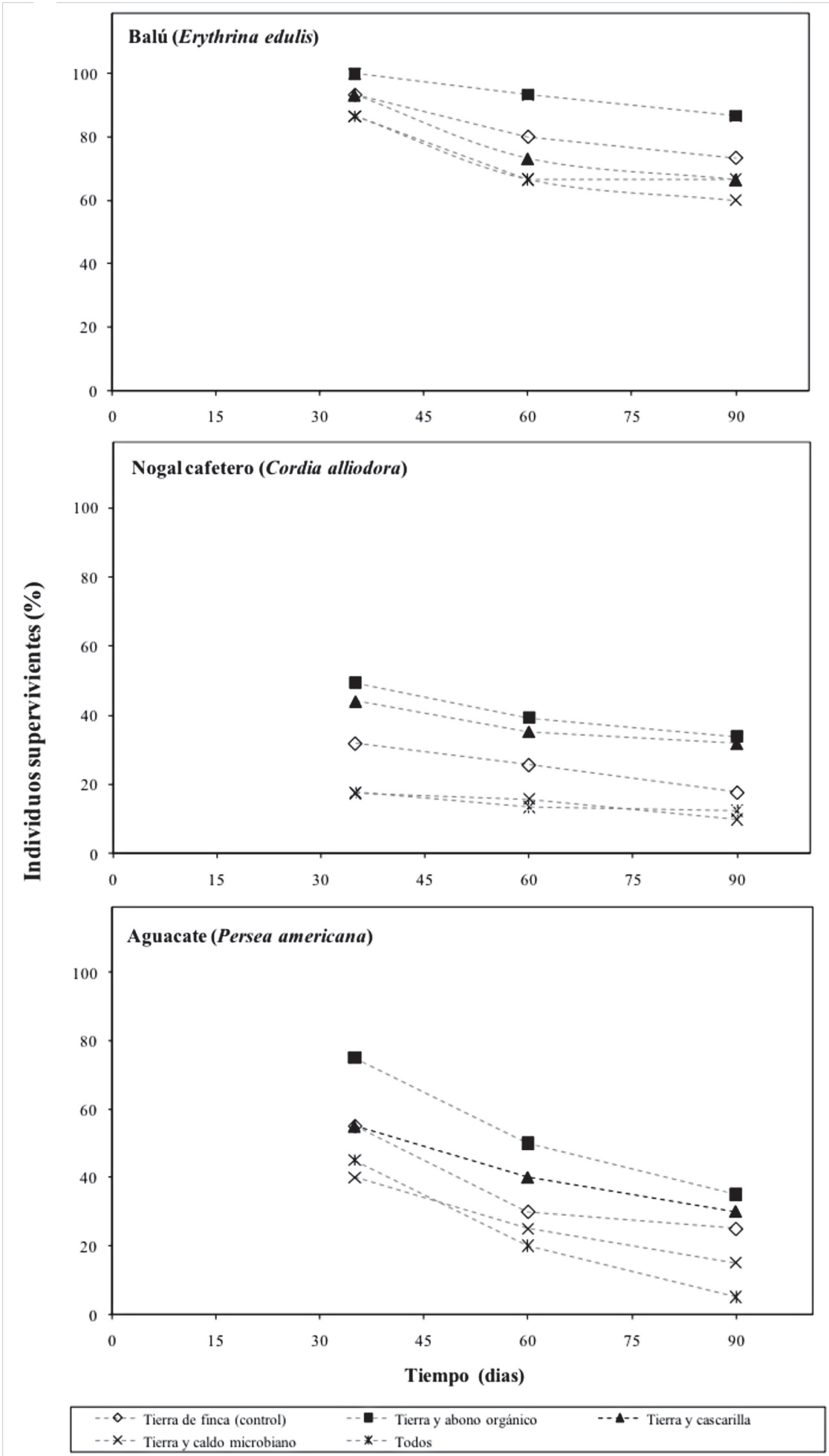

Figura 2. Porcentaje de germinación y supervivencia de semillas del balú (Erythrina edulis $N=75$ ), nogal cafetero (Cordia alliodora $\mathrm{N}=100 \mathrm{~g}$ ) y aguacate (Persea americana $\mathrm{N}=70$ ) durante 90 días desde el establecimiento del ensayo. 


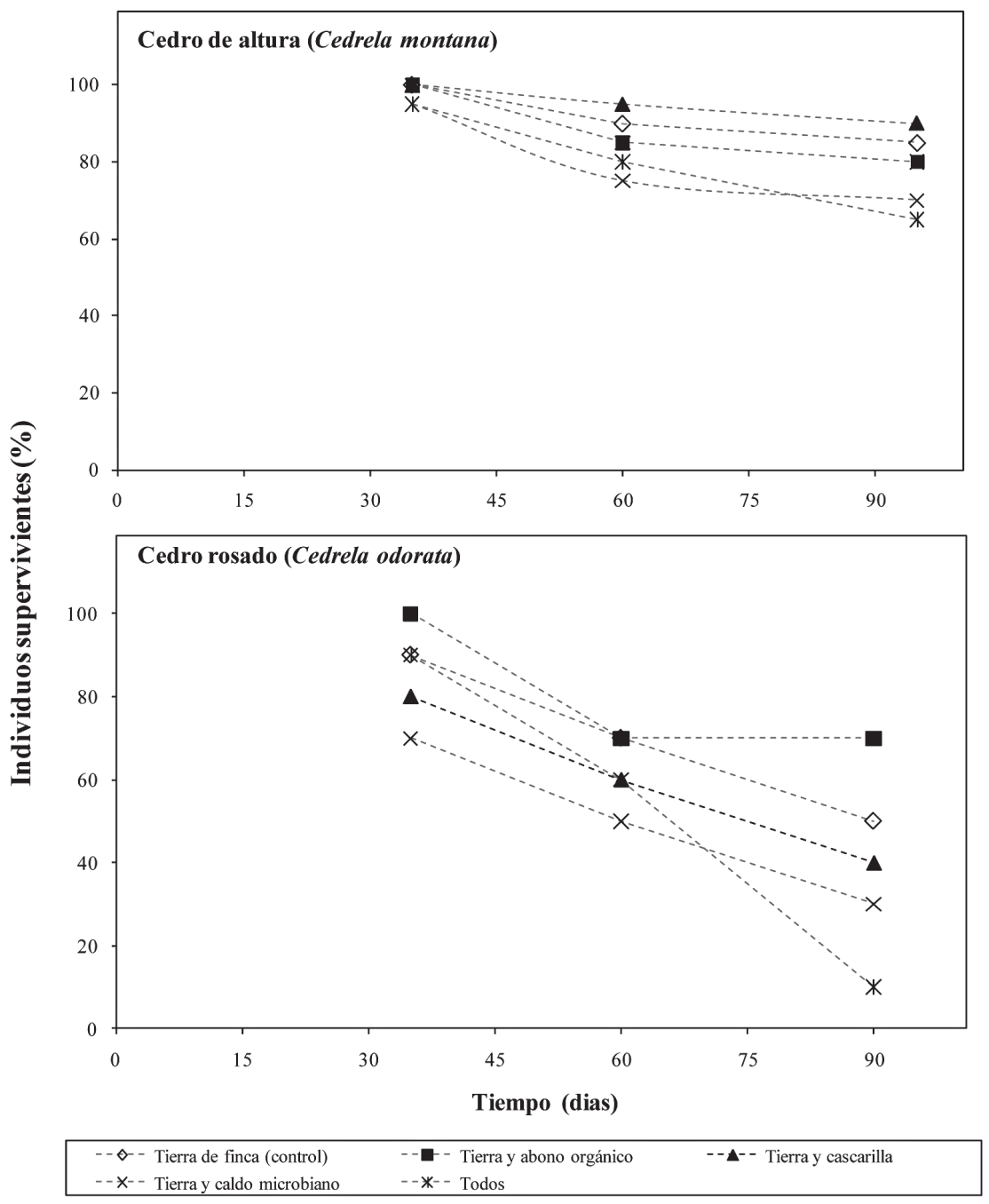

Figura 3. Porcentaje de supervivencia de plántulas rescatadas del bosque (regeneración natural) de cedro de altura (Cedrela montana) y cedro rosado (Cedrela odorata), a lo largo de 90 días a partir del establecimiento del ensayo.

Fuente: elaboración propia 